\title{
Second Order Mutual Information based Grey Wolf Optimization for effective storage and de-duplication
}

\author{
JYOTI MALHOTRA* and JAGDISH BAKAL \\ Department of Computer Science and Engineering, G. H. Raisoni College of Engineering, affiliated to RTM \\ University Nagpur, Nagpur 440016, India \\ e-mail: jyotijmalhotra1@gmail.com; jyoti.j.malhotra@gmail.com; bakaljw@gmail.com
}

MS received 4 October 2017; revised 16 April 2018; accepted 18 April 2018; published online 9 October 2018

\begin{abstract}
This paper intends to perform de-duplication for enhancing the storage optimization by utilizing the similarity in mutual information. Hence, this paper contributes by proposing a hybrid fingerprint extracting using SH and HC algorithms. Secondly, the data is clustered using the latest technique called as SOMI-GO to extract the metadata. The extracted metadata is stored in metadata server which provides better storage optimization and de-duplication. SOMI-GO is adopted as it provides maximum second-order mutual information based on the similarity index. The proposed SOMI-GO technique is compared with the existing methods such as K-means, K-mode, ED-PSO, ED-GA and ED-GWO in terms of accuracy, TPR, TNR and performance time and the significance of the SOMI-GO method is described.
\end{abstract}

Keywords. De-duplication; simhash algorithm; Huffman coding; Grey Wolf Optimization; accuracy.

\section{Introduction}

The increase in the development of data in data centres leads to the issues in de-duplicating files or documents [1]. A report submitted by IDC verifies that in the modern world, data are increasing for every 18 months and it is also known that $75 \%$ of the saved files are being duplicated. Particularly in the backup system, the rate of duplication of data crosses 90\% [2-4]. Hence protecting and optimizing the data is very important as they are increasing in their sizes [5]. Losing of data is also to be concerned. For storing and to enhance the reliability of data, de-duplication techniques are employed widely. Also it is a different method that provides backup data efficiently [6]. Data De-duplication [2] also helps to compress the data and to discard the redundant files or documents [7-11]. Thus it serves as a better agent for storing data and compressing files. It can also be adopted in transferring data by reducing the number of bytes thus enhancing storage optimization. The chunks or patterns of bytes that share similar information are identified and stored for verification purpose [12-16].

De-duplication alleviates the possibility of data loss by reducing storage footprints (presumptuous, which data loss events equally happen across the entire disk space) [17-19]. Conversely, it amplifies the severity of each data loss event that might corrupt multiple chunks or files, which share the same lost data $[20,21]$. Numerous studies in the literature have addressed de-duplication storage reliability in

*For correspondence different ways. For instance, some studies (e.g., [22, 23]) add redundancy through replication or erasure coding to post-de-duplication data for fault tolerance [24]. Other studies (e.g., [25]) propose quantitative approaches to assess de-duplication storage reliability. On the other hand, there remain two key open reliability issues that are further complicated by the data sharing nature of de-duplication [26-28].

De-duplication technique is not only implemented for data that are structured, but they also store key-words, backup systems and file systems that are distributed [29]. as many of the cloud environments are duplicated. Anyhow, many methods of de-duplication are implemented for storage efficiency only. DupLESS [21] is a technique that provides good privacy strategies and solution for the problem of CE scheme. CDC [30] scheme is a method employed to de-duplicate the chunks and avoid the shifting issues of boundaries. RMD [5] and inline de-duplication model named I-sieve [6] are adopted as they respond quickly for any problems arising in fingerprint extraction [31-33].

De-duplicating data are also subjected to some problems. The main issue faced by them are due to the intensities based on computational resource [29, 34-37]. As the integration of data depends on the design of de-duplication system, it also causes the problem to these techniques [38]. Scaling is another obstacle raised due to the sharing of scope between the storing devices. Also it is necessary to balance the quality of data while the de-duplicating process takes place. If there is more backup equipment, the 
efficiency of space is also affected $[39,40]$. Further, there is a possibility of the data being attacked by hackers if the hash values are guessed or known by them. But overall, there are many strategies for de-duplicating files, but still it is in need for an enhanced de-duplicating method with storage optimization [41-43, 54].

The main contribution of this paper is to introduce a hybrid fingerprint extracting using $\mathrm{SH}$ and $\mathrm{HC}$ algorithms. Then, the data is clustered by SOMI-GO in order to extract the metadata. The extracted metadata is stored in metadata server that presents better storage optimization and de-duplication. SOMI-GO is adopted as it provides maximum second-order mutual information on the basis of the similarity index.

This paper is structured as follows: initially, the related works are studied in section 2 . In section 3 , the description of the Fingerprint Clustering and Metadata Construction is provided. The Fingerprint Clustering De-Duplication Architecture is discussed in section 4. In section 5, the simulation results are presented. Finally, the main concluding remarks are reported in section 6 .

\section{Related works}

In 2015, Xing et al [2] had presented a de-duplication system named AR-De-dupe to cluster and enhance the efficiency and reliability of storing data. De-duplication methods confront some issues due to the overhead of communication for routing data, reduction in the rate of deduplication due to increasing number of servers and load balance for enhancing throughput. The application-aware mechanism is used in routing server to fasten the handprint indexes. The implemented technique is analyzed in 2 data sets. The results also describe the ability to obtain a high rate of de-duplication. Reduction in overhead communication and better load balance are achieved by the use of a data routing algorithm. Also the application aware mechanism provides $30 \%$ better performance.

In 2016, Zhang et al [44] have proposed a new CDC algorithm for de-duplication in chunk level named AE code. This method has eliminated the issues caused by moving the extreme values. The experiments have been verified by utilizing real-world datasets and an increase in throughput is achieved. This method can find the correct boundaries of chunks and it gives chunks with small variance. The AE scheme enhances the throughput of CDC algorithms. The throughput of the system has increased to $50 \%$ and it provides 2.3 larger improvements than other systems.

I-sieve is a mechanism introduced in 2015 by Wang et al [6]. It is aimed to obtain high data sieve performance based on iSCSI. Also a cache of multilevel is used to minimize the utility of RAM and it optimizes the performance of lookup and index tables. Depending on the iSCSI with open source, an I-sieve prototype is introduced. The experiments are done by testing virtual images and tools. The obtained results show that the proposed method can aid and improve the foreground enhancement. The I-sieve technique co-exists with the other conventional methods as it includes the iSCSI protocol.

\section{Fingerprint clustering and metadata construction}

Let $\mathrm{F}$ be the fingerprint of files extracted from $\mathrm{SH}$ and $\mathrm{HC}$ algorithms. The fingerprint extracted from $\mathrm{SH}$ is given by, $F_{i j}^{S H}$ and the fingerprints obtained from $\mathrm{HC}$ is represented by, $F_{i j}^{H C}$.

Metadata is the representative of chunk groups to be denoted as a fingerprint. For metadata construction, the chunks are classified into two groups namely, homogeneous chunks and heterogeneous chunks. The chunks that hold somewhat similar keywords are said to be homogenous chunks and those chunks which do not share similar keywords, i.e., the keywords are very much differed from each other is known as heterogeneous chunks. The clustering process involved in this paper is based on the Euclidean distance. K-means [44], SOMs [29] etc. are certain algorithms used for clustering but due to lack of dimensionalities and complexities, GWO technique [45] is used in this paper for clustering process.

Equation (1) gives the model for clustering the chunks, where $Q_{c}^{*}$ is the centroid which is optimum. All the individual clusters are addressed by a centroid, which is shown by $Q_{c}: c=1,2, \ldots, N_{c}$. Chunks that represent the respective cluster also represent the centroid that is addressed by chunks. Equation (1) reduces the errors in clustering, and so the metadata can be constructed without errors.

$$
\begin{gathered}
Q_{c}^{*}=\arg \max _{Q_{c}}\|S O M I\| \\
\sum_{c, c^{\prime}} \sum_{d, d^{\prime}} P_{c d} \log \left(\frac{P_{c d}}{P_{c} P_{d}}\right) .
\end{gathered}
$$

\subsection{SoMI- based similarity function}

Each centroid consists of its corresponding values for $\mathrm{SH}$ and $\mathrm{HC}$ fingerprints. By taking probability function for each set of the centroid, the value of $P_{c}$ can be found out. On considering $C_{1}$, the probability of $P_{c 1}$ can be obtained. Likewise the probabilities of $n$ number of centroids can be found out. Similarly, the data consists of respective values of SH and HC fingerprints. The value $P_{d 1}$ can be obtained by taking probability function for the first set of data. Similarly probabilities are taken for all sets of data. The values of joint probability between centroid and data point $P_{c d}$ can be obtained by taking probabilities for both $P_{c}$ and $P_{d}$, i.e., centroid probability and data probability. SOMI can be achieved by comparing the probabilities of $P_{c}, P_{d}$ and $P_{c d}$. 
Thus by comparing the probability of each centroid with all sets of data probabilities, the SOMI's can be obtained using Eq. (2) The achieved SOMI values are then compared and the SOMI, which shares more mutual information is selected and the data are allotted in the centroids correspondingly. The maximum MI is represented as $S O M I_{\max }$, which is desired to be achieved and then they are stored in centroid represented by $Q_{c}$. The pseudo code to extract the fingerprints from chunks is explained by algorithm 1 .

\subsection{EDGWO based clustering}

Algorithm 1: Pseudo code for SOMI-GO to extract the fingerprint from chunks

Step 1 Assign the population of the grey wolf as $X_{i}$, where $i$ lies between 1 to $n$

Step 2 Assign values for $a, A$ and $C$

Step 3 Each search agent is calculated by its fitness

Step 4 While (maximum number of iterations $>\mathrm{t}$ )

$$
\text { Select } X_{\alpha} X_{\beta} \text { and } X_{\gamma}
$$

Step 5 For each and every search agents

The position of the current search agent is updated by end for using Eq.(1)

$a, A$ and $C$ are updated.

Measure the fitness of all the wolves

The iterations are increased by 1, i.e.

$t=t+1$

End while

Return $X_{\alpha}$.

Using Eq. (1), the objective model can be achieved by GWO [21] algorithm employed here. This algorithm explains the hunting nature of grey wolves. The grey wolves are classified in to $\alpha, \beta, \gamma, \delta$, based on their hierarchy of hunting. Initially the wolves search for prey and then it encircles the prey and then attacks it. These are the three things which are adopted to improve the optimization process (figure 1).

Grey wolves address the optimal value where the wolves are bounded by a number which is random in nature. i.e., $Q_{c}\left(S O M I_{\max }\right)=\left[Q^{\min }, Q^{\max }\right], \quad$ where, $\quad Q^{\min }=\min \left(F_{i j}^{D}\right)$ and $Q^{\max }=\max \left(F_{i j}^{D}\right)$, respectively and $F_{i j}^{D}$ is the decimal version of $F_{i j}$.

Till the best wolf is identified by using SOMI based evaluation process, the iteration continues.

Using Eq. (1), the evaluation takes place for centroids which are initialized. Our target is to find the SOMI with maximum values; hence we assign wolves with $\alpha, \beta, \gamma, \delta$ functions. The centroid with maximum SOMI is assigned as $\alpha$, the one with second maximum SOMI is called $\beta$ and the centroid with third maximum SOMI is said to be $\gamma$. Other wolves that are remaining are called $\delta$. Thus the relationship among the wolves can be addressed by $Q_{E}(\alpha)>Q_{E}(\beta)>Q_{E}(\gamma)>Q_{E}(\delta)$.

The fitness values of grey wolves are measured after they are being initialized and the wolves are selected namely, $\alpha, \beta, \gamma$. The particular distance of the wolves with respect to the prey is updated respectively for the number of iterations held. The iterations take place till the wolves with maximum values of SOMI's are obtained. By measuring the distance of $\alpha, \beta, \gamma$, the centroid with optimum value can be achieved using Eqs. (7), (8) and (9). From Eqs. (4), (5) and (6), the mean values of centroids are calculated and then they are updated to updating formula form Eq. (3). The total number of iterations taking place is denoted by $t$.

$$
\begin{aligned}
Q_{c}(t+1)=\exp \left(\frac{t}{t_{\max }}\right) \frac{Q_{c 1}+Q_{c 2}+Q_{c 3}}{3} \frac{1}{\exp \left(t_{\max }\right)} \\
Q_{c 1}=Q_{c \alpha}-A_{1}\left(D_{\alpha}\right) \\
Q_{c 2}=Q_{c \beta}-A_{2}\left(D_{\beta}\right), \\
Q_{c 3}=Q_{c \delta}-A_{3}\left(D_{\delta}\right) \\
D_{\alpha}=\left|C_{1} \cdot Q_{c \alpha}-Q_{c}\right| \\
D_{\beta}=\left|C_{2} \cdot Q_{c \beta}-Q_{c}\right| \\
D_{\delta}=\left|C_{3} \cdot Q_{c \delta}-Q_{c}\right| \\
C=2 \cdot r_{2} \\
A=2 a \cdot r_{1}-a
\end{aligned}
$$

$A$ and $C$ represents the coefficient vectors as given in Eqs. (10) and (11) and the random vectors are denoted by $r_{1}$ and $r_{2}$. By changing $A$ and $C$ values of the vector, the best locations of the wolves can be identified according to the current position. Any location that lies between the iterations can be reached by getting the permission from $r_{1}$ and $r_{2}$. The centroids that are updated using Eq. (3) are further updated using Eq. (12), where $Q_{c p}$ denotes the position of the prey.

$$
\begin{gathered}
Q_{c}(t+1)=Q_{c p}(t)-A . D \\
D=\left|C \cdot Q_{c p}(t)-Q_{c}(t)\right|
\end{gathered}
$$

\subsection{Metadata construction}

The location of prey can be found out after performing a number of iterations using $\alpha, \beta$ and $\gamma$. All the solutions are 


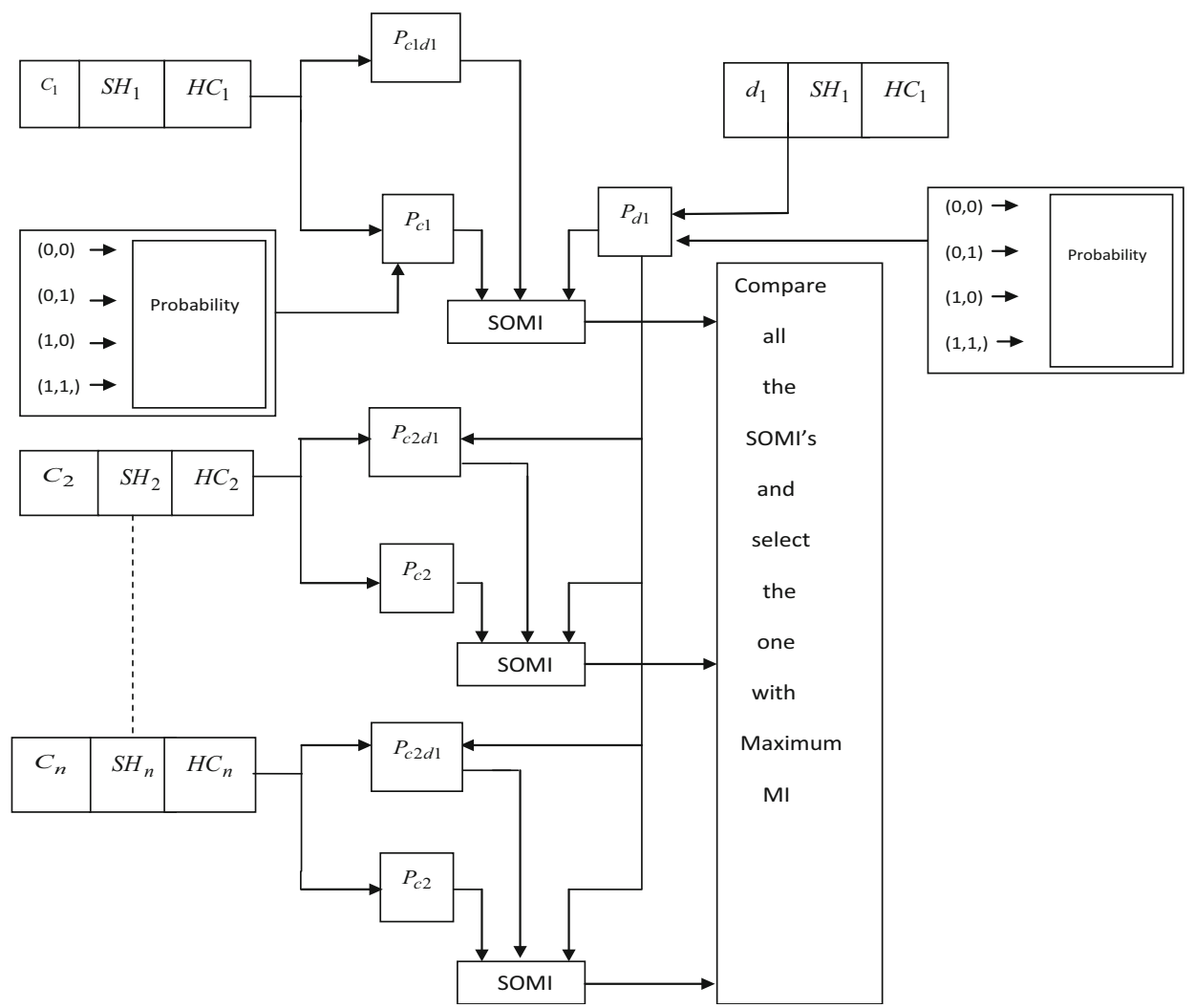

Figure 1. Fingerprint extraction based on ED-GWO clustering.

evaluated using Eq. (1) updated using proposed updating model, which is described in Eq. (3). The prey which is updated with maximum SOMI is considered as the best wolf. Thus by utilizing GWO technique, better SOMI can be achieved. $\alpha$ is the best wolf with maximum $S O M I_{\max }$. The metadata server saves the centroid as metadata and then clustering is performed. The clustered data are then indexed and saved in index library and the storage disk saves the indexed data.

\section{Fingerprint clustering de-duplication architecture}

Online and offline storage are the two processes involved in the storage of metadata. In offline mode, if a document or webpage is given as input, the partition of chunks takes place primarily. Then the partitioned chunks are subjected to $\mathrm{HC}$ and $\mathrm{SH}$ for identification and detection of nearer duplicates. The fingerprints extracted using $\mathrm{SH}$ and $\mathrm{HC}$ algorithms are then concatenated properly. SOMI-GO technique is adopted for clustering process. The centroids obtained from clustering is said to be the metadata which is stored on the metadata server. The index table stores the corresponding chunk of the index. Meanwhile after indexing, the chunks are stored in a storage disk (figure 2).

Similarly, in online mode, the chunks are partitioned initially when a new file has to be saved. Therefore new chunks are created for data that do not share similar contents and then they are stored on a storage disk. Metadata server stores the newly generated metadata. Then the index table stores the indices after being formulated.

\subsection{Fingerprint extraction}

$\mathrm{SH}$ algorithm is used for computing fingerprints to detect the closer duplicates [46]. SH has 2 important but conflicting properties that make it ideal to adopt for detecting the availability of nearer duplicates. They are (1) the hash properties are defined by a fingerprint and (2) hash includes similar with same values. Thus it helps in determining the similarity of chunks by distinguishing their hash values, respectively. Initially each chunk is converted and a set of keywords are filtered. Weights are assigned to each 


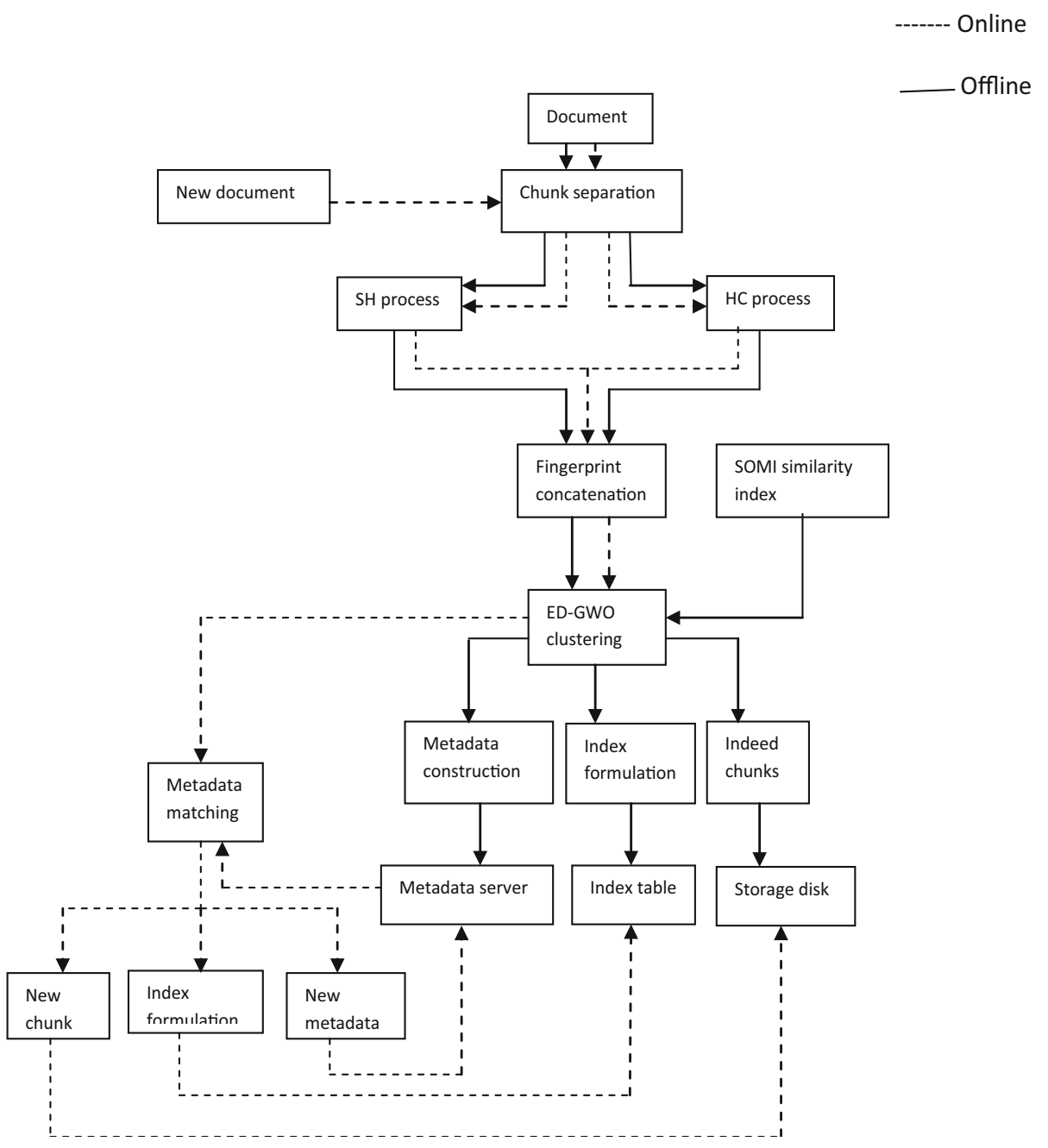

Figure 2. Systematic representation of de-duplication for storing files.

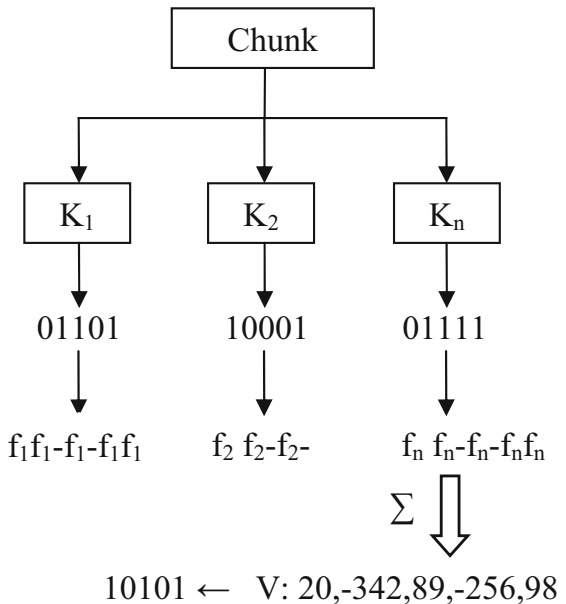

Figure 3. Generation of fingerprints for file chunks using $\mathrm{SH}$. keyword correspondingly, i.e., the total appearance of $C_{i j}$ in keywords. A HD vector is generated by converting the f-bit fingerprint. The value of ' $f$ ' is smaller than its original dimension.

Consider the input " $C_{i j}$ " which is pre-processed and includes a set of keywords. Zero is assigned to all the elements in f-dimensional vector V. After the above process, the f-bit values are generated from each keyword " $C_{i j}$ ". The number of f-bits may decrease or increase depending on the weights and the number of keywords. The signs of the components address the certain bits of last fingerprint of " $C_{i j}$ ". The extraction of fingerprints takes place after repeating the process for each individual " $C_{i j}$ ".

The fingerprints of chunks are given by $F_{i j}^{S H}$ in this $\mathrm{SH}$ algorithm. The pseudo code of the $\mathrm{SH}$ algorithm is given in Algorithm 1 and the detailed process is given in figure 3 . 


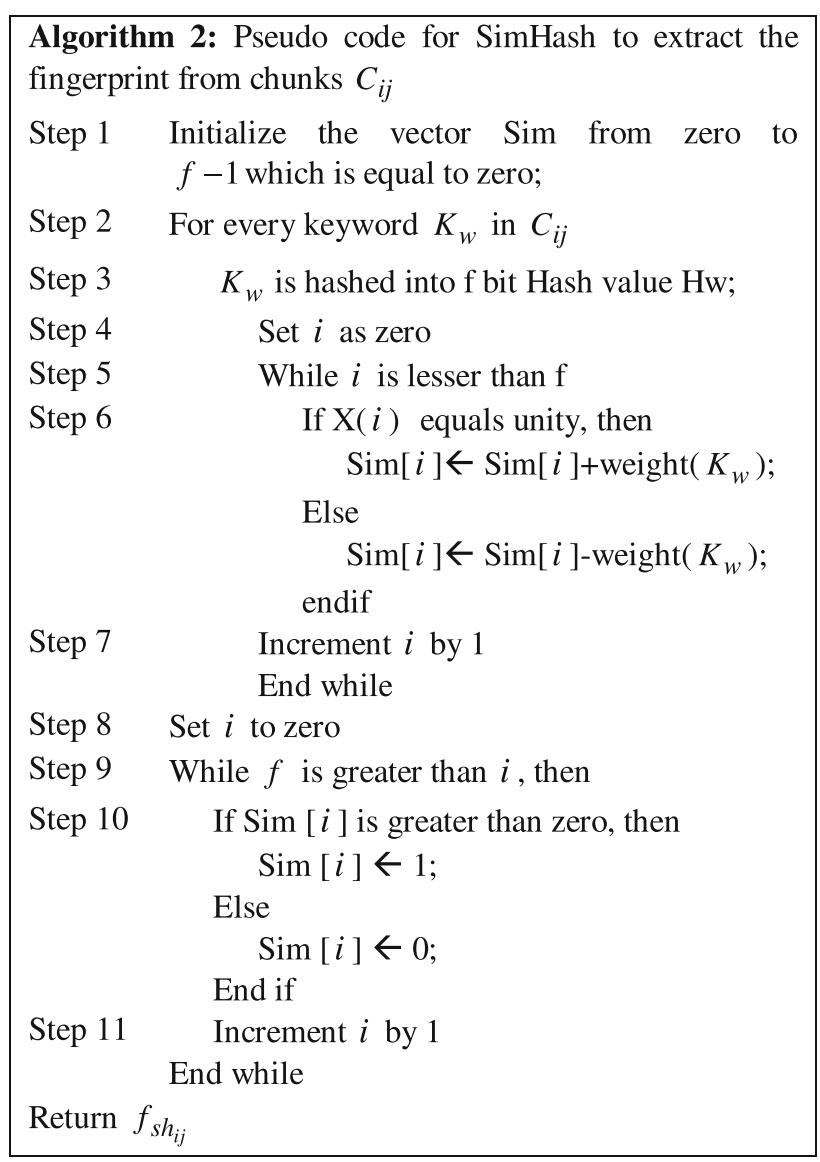

\subsection{HC fingerprint}

SH coding is a new technology which is being used for fingerprint extraction from chunks. As the fingerprint based on SH concentrates mainly on keyword indexing, the method proposed in this paper also includes fingerprints based on $\mathrm{HC}$ [47] and produces the final fingerprints by utilizing both $\mathrm{SH}$ and $\mathrm{HC}$. $\mathrm{HC}$ dependent fingerprints mostly concentrate on frequency, i.e., it produces fingerprints depending on the total number of keyword appearance. It does not focus mainly on indexing. Thus HC algorithm provides better partitioning of chunks and deduplication based on the frequency of chunks.

Using various weights and frequencies, a tree is built up. $K_{1}$ is assigned with an associated frequency of $7, K_{2}$ with 6 , $K_{3}$ with a frequency $5, K_{4}$ with a relative frequency of 3 and $K_{5}$ with an associated frequency of 2 .

The respective symbol for all the nodes can be found out by starting from the root and moving down till the leaf with the corresponding symbol is reached. For all the branches in the left side, zeros are assigned and for all the right side branches, one's are assigned branch. The HC for entire keywords is given as, 01011011101111 . The fingerprints of

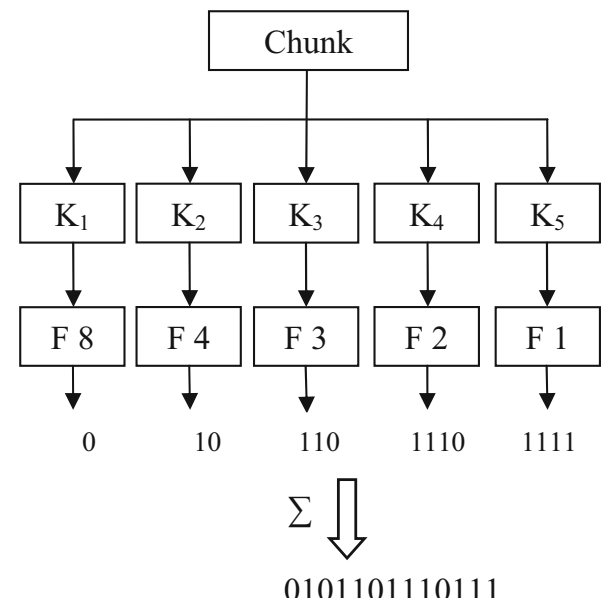

Figure 4. Generation of fingerprint for file chunks using HC.

$\mathrm{HC}$ are represented by $F_{i j}^{H C}$ (figure 4). The whole fingerprint can be represented by, $\left|F_{i j}\right|=\left|F_{i j}^{S H}\right|+\left|F_{i j}^{H C}\right|$.

\section{Simulation results}

\subsection{Simulation}

The proposed intelligent de-duplication methodology is developed in JAVA and compared with traditional methods such as PSO, GA and GWO [48-50], which are used for clustering. Moreover, the traditional K-Means [45] and K-Mode [51] clustering algorithms are also used for comparative study. Henceforth, the proposed method is called here as SOMI-GO, ED-PSO [52] and ED-GA [53]. The benchmark database, called 20 Newsgroups dataset (which can be accessed from http://qwone.com/ jason/20News groups/) is synthesized for duplication by $20 \%, 30 \%, 40 \%$ and $50 \%$. The comparisons on identifying and reconstructing from duplication are studied using accuracy, TPR and TNR rates against the different percentage of duplication.

From figure 5a, it can be seen that all the methods increase with respect to increase in restoration accuracy. The proposed method is better than ED-GWO by $7 \%$, EDPSO by $5.5 \%$, K-mode by $2.7 \%$, K-means by $4 \%$ and EDGWO by $3.2 \%$. Thus it can be known that the proposed method provides better restoration accuracy when compared with the existing techniques. From figure $5 b$, it is observed that all the techniques increase with an increase in restoration accuracy. However, the proposed method is better than ED-GA by $8 \%$, ED-PSO by $6.8 \%, \mathrm{~K}$-mode by $3.2 \%$, K-means by $4.2 \%$ and ED-GWO by $4.6 \%$. Thus the capability of the proposed method to exhibit better 


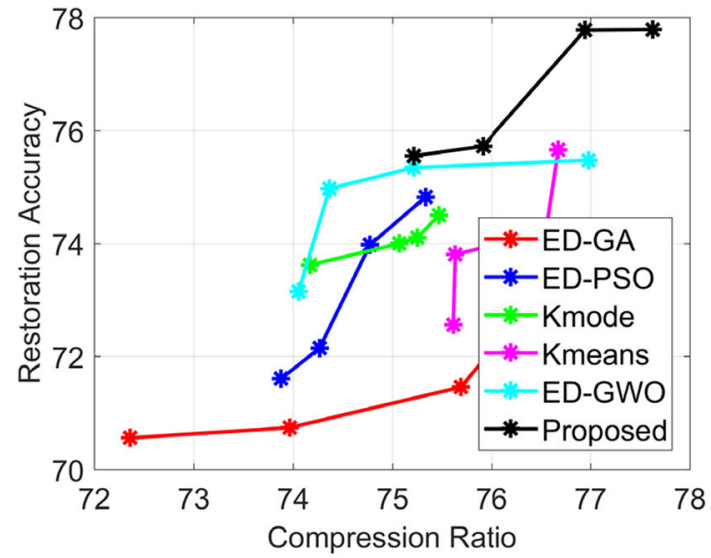

(a)

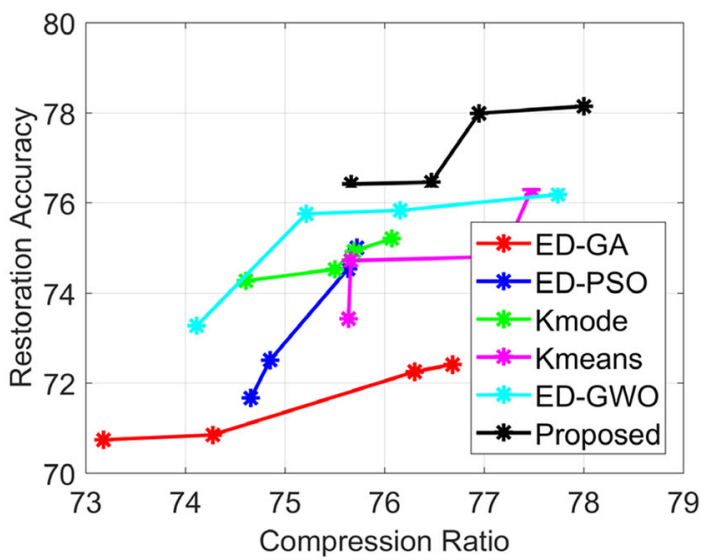

(b)

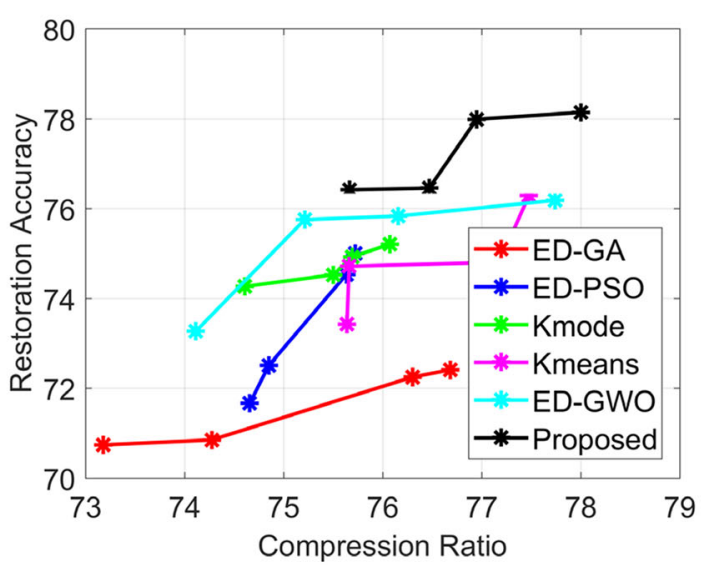

(c)

Figure 5. Analysis of the proposed and existing method of the restoration accuracy and compression ratio (a) first iteration, (b) second iteration and (c) third iteration.

accuracy is well-established. From figure 5c, it is noted that all the methods increase with respect to increase in restoration accuracy. However, the proposed method is better than ED-GA by $8 \%$, ED-PSO by $6.8 \%$, K-mode by $3.2 \%$, K-means by $4.2 \%$ and ED-GWO by $4.6 \%$. Thus the ability of the proposed method to exhibit enhanced accuracy is verified.

From figure $6 \mathrm{a}$, it can be seen that the methods are iterated for $20 \%$ duplication ratio. All the methods increase with respect to increase in accuracy. The proposed method is proved to be better than ED-GA by $0.85 \%$, ED-PSO by $0.7 \%$, K-means by $0.5 \%, \mathrm{~K}$-mode by $0.4 \%$ and ED-GWO by $0.2 \%$. Hence it is showed that the restoration accuracy in terms of maximum iterations is better in the proposed method than the conventional methods. The existing and the proposed methods are iterated for $30 \%$ of duplication rate in figure $6 \mathrm{~b}$. The entire methods go on increasing with respect to increasing in restoration accuracy. The ED-GA method is better than the proposed technique by $0.9 \%$, EDPSO by $0.85 \%$, K-mode by $0.7 \%$, K-means by $0.7 \%$ and ED-GWO by $0.5 \%$, thus showing the enhancement of the proposed method. From figure $6 \mathrm{c}$, it can be noted that all the methods are increasing with an increase in accuracy. However, the implemented method is better than ED-PSO by $0.7 \%$, K-mode by $0.7 \%$, ED-GWO by $0.5 \%$, ED-GA by $0.98 \%$ and $\mathrm{K}$-means by $0.7 \%$. Thus it is verified that the proposed technique can deliver better restoration accuracy than the existing techniques. From figure $6 \mathrm{~d}$, it is known that the entire methods increase with respect to increase in restoration accuracy. The implemented technique is better than ED-GWO by $0.42 \%$, ED-GA by $0.84 \%$, ED-PSO by $0.5 \%$, K-means by $0.42 \%$ and $\mathrm{K}$-mode by $0.7 \%$, thus enabling the better performance of the proposed method.

The TNR for $20 \%$ duplication ratio is given by figure $7 \mathrm{a}$ and it can be observed that all the methods are increasing with respect to increasing in TNR values. The proposed 


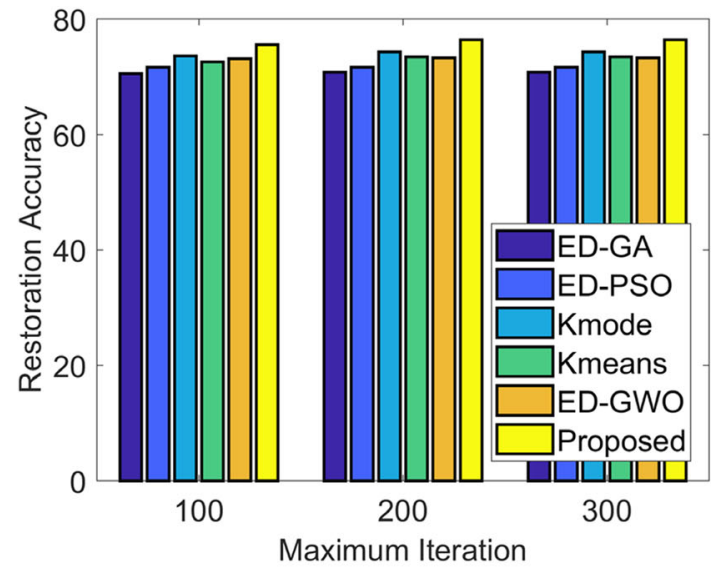

(a)

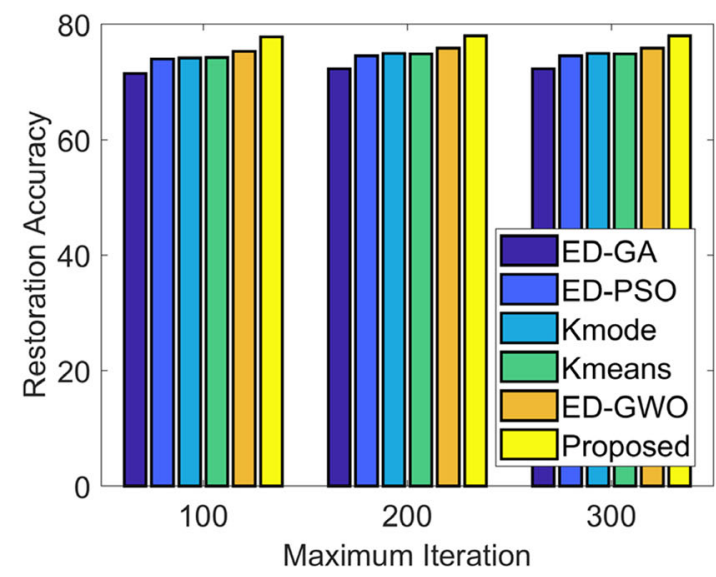

(c)

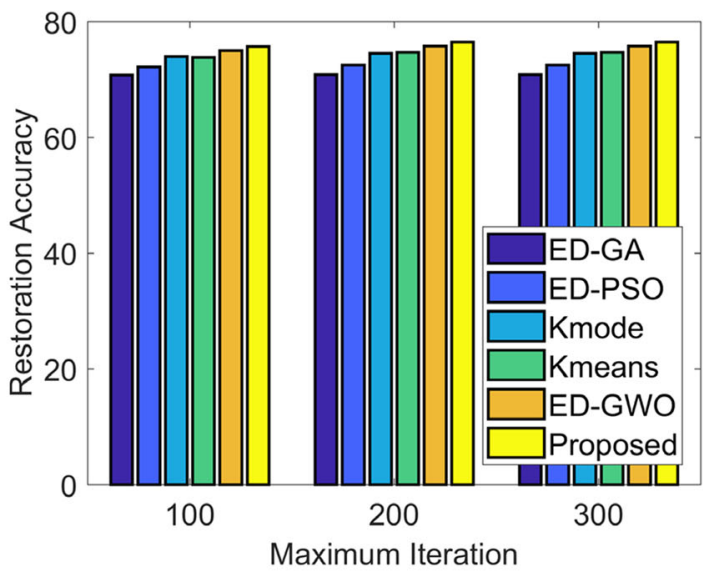

(b)

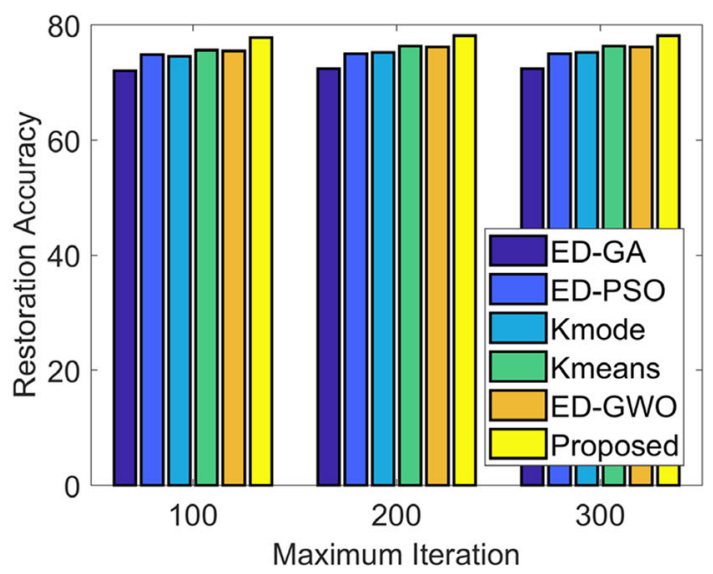

(d)

Figure 6. Analysis of the proposed and existing method of restoration accuracy and maximum iteration (a) 20\% duplication rate, (b) $30 \%$ duplication rate, (c) $40 \%$ duplication rate, and (d) $50 \%$ duplication rate.

method is better than ED-GA by $7.1 \%$, ED-PSO by $1.4 \%$, $\mathrm{K}$-mode by $2.8 \%$, K-means by $1.4 \%$ and ED-GWO by $0.7 \%$, thus showing the improved version of the implemented method. The TNR for $30 \%$ duplication ratio is provided in figure $7 \mathrm{~b}$ and it can be noticed that all the techniques are increasing with increase in TNR values. The proposed method is far better than ED-GA by $5.5 \%$, EDPSO by $5.5 \%$, K-mode by $4.1 \%$ and K-means by $2.7 \%$ and ED-GWO by $4.1 \%$, thus showing the enhancement of the implemented method. The TNR for $40 \%$ duplication ratio is given by figure $7 \mathrm{c}$. It can be known that all the techniques raise with respect to increase in TNR values. The proposed method is better than ED-GA by $4.1 \%$, ED-PSO by $5.5 \%$, $\mathrm{K}$-mode by $4.1 \%$, K-means by $2.7 \%$ and ED-GWO by $4.1 \%$. This evaluation shows the better performance ability of the proposed method. The TNR for $50 \%$ duplication ratio is given by figure $7 \mathrm{~d}$ and it can be observed that all the methods increases with respect to TNR values, however the proposed method is better than ED-GA by $5.4 \%$, ED-PSO by $4.7 \%$, K-mode by $6.8 \%$, K-means by $4.1 \%$ and EDGWO by $2.7 \%$, thus showing the improved capability of the implemented method.

The TPR for $20 \%$ duplication ratio is given by figure $8 \mathrm{a}$ and it can be observed that all the methods are increasing with respect to increasing in TPR values. The proposed method is better than ED-GA by $5.06 \%$, ED-PSO by $3.7 \%$, $\mathrm{K}$-mode by $2.5 \%$, K-means by $2.5 \%$ and ED-GWO by $1.2 \%$, thus showing the improved version of the implemented method.

The TPR for $30 \%$ duplication ratio is provided by figure $8 \mathrm{~b}$ and it can be noticed that all the techniques are increasing with increase in TPR values. The proposed 


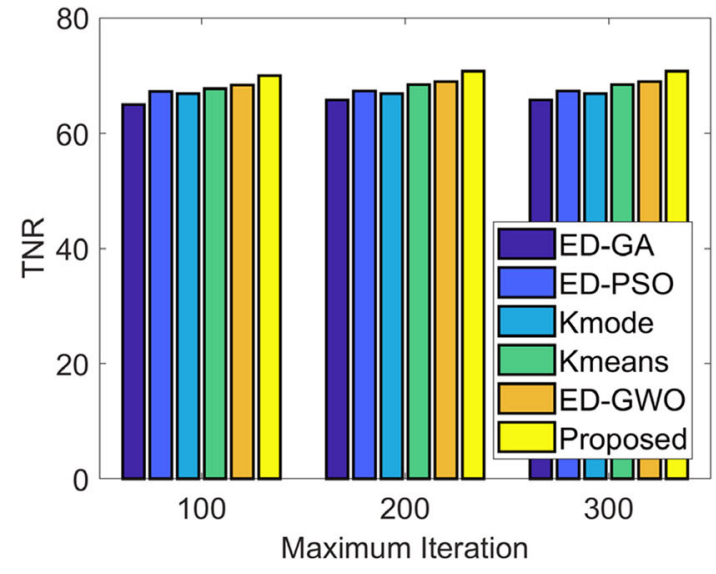

(a)

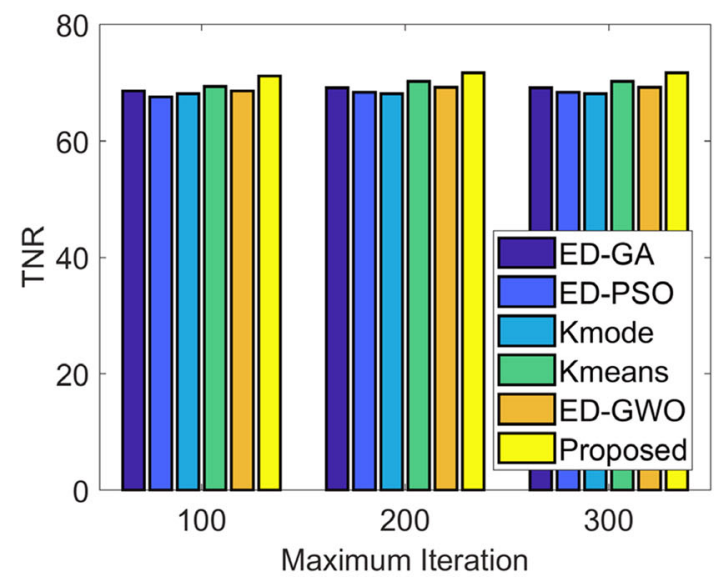

(c)

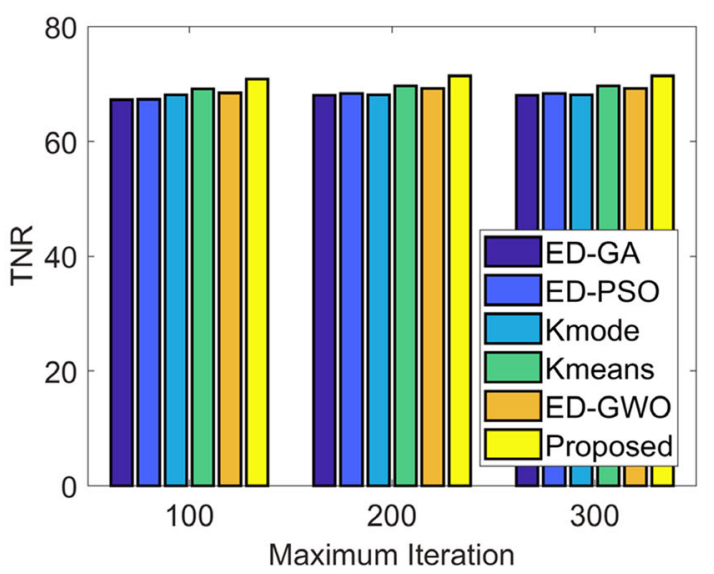

(b)

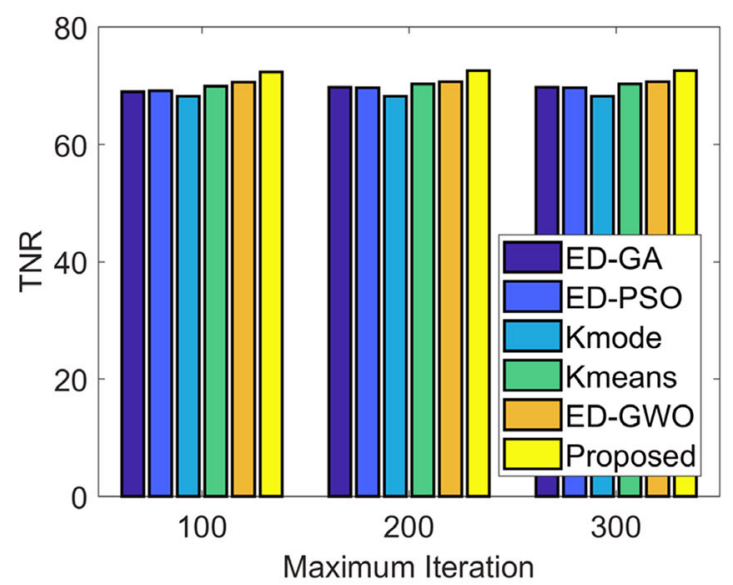

(d)

Figure 7. Analysis of the proposed and existing method of TNR and maximum iteration (a) $20 \%$ duplication rate, (b) $30 \%$ duplication rate, (c) $40 \%$ duplication rate, and (d) $50 \%$ duplication rate.

method is far better than ED-GA by $5.06 \%$, ED-PSO by $3.7 \%$, K-mode by $2.5 \%$ and $\mathrm{K}$-means by 2.5 , thus showing the enhancement of the implemented method.

The TPR for $40 \%$ duplication ratio is given in figure $8 \mathrm{c}$. It can be known that all the techniques raise with respect to increase in TPR values. The proposed method is better than ED-GA by $4.9 \%$, ED-PSO by $3.7 \%$, K-mode by $2.4 \%$, $\mathrm{K}$-means by $2.4 \%$ and ED-GWO by $2.4 \%$. This evaluation shows the better performance ability of the proposed method. The TPR for 50\% duplication ratio is given in figure $8 \mathrm{~d}$ and it can be observed that all the methods increases with respect to TPR values, however the proposed method is better than ED-GA by $4.8 \%$, ED-PSO by $2.4 \%$, $\mathrm{K}$-mode by $3.6 \%$, K-means by $2.4 \%$ and ED-GWO by $2.4 \%$, thus showing the improved capability of the implemented method.
From table 1, it is noted that all the techniques are increasing with increase in the percentage of duplication. Anyhow, the proposed method is better than K-mode by $1.4 \%$, ED-PSO by $1.4 \%$, K-means by $0.15 \%$, ED-GA by $3.4 \%$ and ED-GWO by $1.2 \%$. Thus it is proved that the compression ratio for the implemented technique is much better than the existing methods.

From table 2, it can be seen that the normalized computing time is increased with increase in duplication ratio. The computation time for the proposed method is slightly greater than the existing methods, but the accuracy of the implemented method is much larger than the ratio of the computation time. The proposed method is better than EDGA by $8 \%$, ED-PSO by $6.8 \%$, K-mode by $3.2 \%$, K-means by $4.2 \%$ and ED-GWO by $4.6 \%$ in terms of accuracy and 


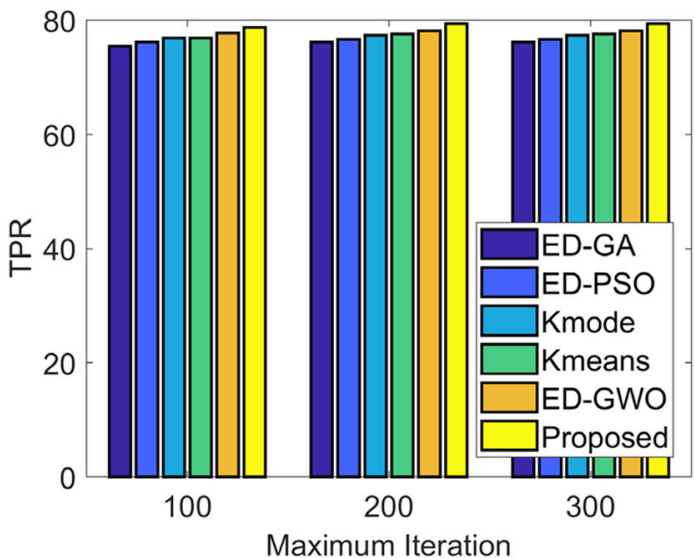

(a)

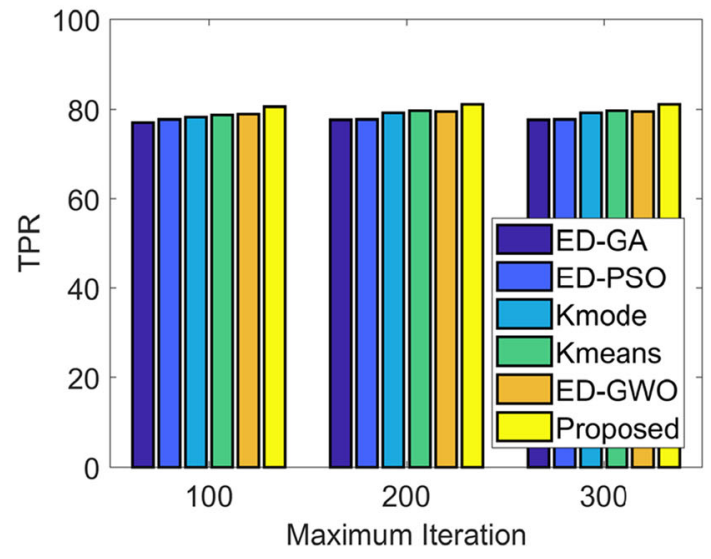

(c)

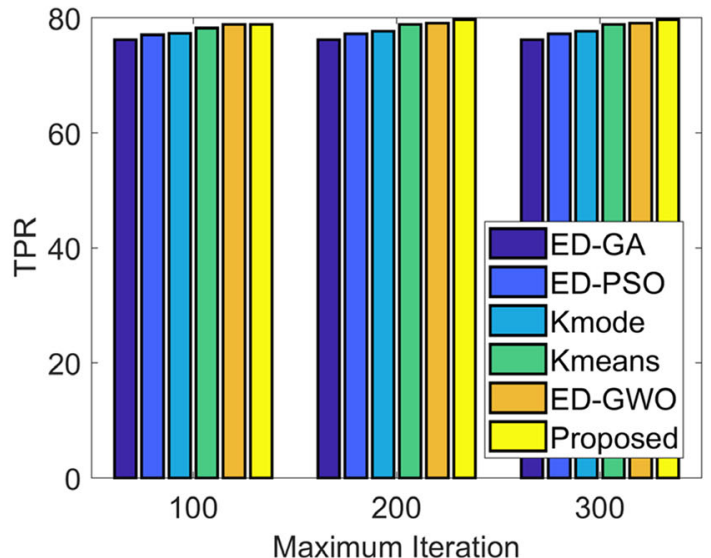

(b)

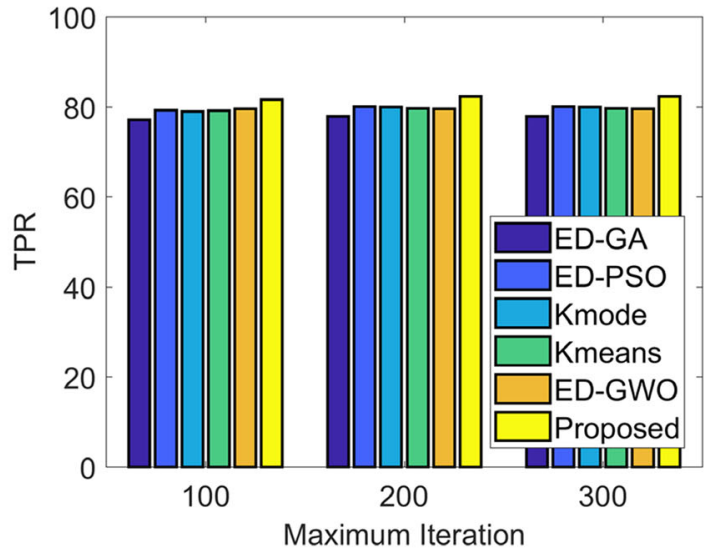

(d)

Figure 8. Analysis of the proposed and existing method of TPR and maximum iteration (a) $20 \%$ duplication rate, (b) $30 \%$ duplication rate, (c) $40 \%$ duplication rate, and (d) $50 \%$ duplication rate.

Table 1. Comparison analysis of the proposed and existing methods in terms of compression ratio.

\begin{tabular}{cccccc}
\hline $\begin{array}{l}\text { Percentage of } \\
\text { duplication }\end{array}$ & K-mode & ED-PSO & K-means & ED-GA & ED-GWO \\
\hline 20 & 74.463 & 74.399 & 75.63 & 72.907 & 74.094 \\
30 & 75.358 & 74.655 & 75.652 & 74.173 & 74.931 \\
40 & 75.545 & 75.348 & 76.97 & 76.098 & 75.844 \\
50 & 75.871 & 75.592 & 77.209 & 76.447 & 77.488 \\
\hline
\end{tabular}

Table 2. Normalized computation time of proposed mode and the existing modes.

\begin{tabular}{ccccccc}
\hline $\begin{array}{l}\text { Percentage of } \\
\text { duplication }\end{array}$ & K-mode (s) & ED-PSO (s) & K-means (s) & ED-GA (s) & ED-GWO (s) & Proposed (s) \\
\hline 20 & 0.62 & 0.64 & 0.61 & 0.63 & 0.68 & 0.75 \\
30 & 0.72 & 0.76 & 0.73 & 0.74 & 0.87 & 1 \\
40 & 0.85 & 0.87 & 0.85 & 1 & 0.74 \\
50 & 1 & 1 & 1 & & 1 \\
\hline
\end{tabular}


hence the performance time of proposed method can be considered as negligible.

\section{Conclusion}

In this paper, we have introduced a novel clustering method, SOMI-GO to obtain de-duplication. It overcomes the performance gaps from conventional methods such as, ED-PSO, ED-GA, K-means, K-mode and ED-GWO. Finally, the proposed method SOMI-GO was compared with other existing methods such as K-means, K-mode, ED-PSO, ED-GWO and ED-GA. From the result it was concluded that the proposed de-duplication system provides better TPR of $5.06 \%$, TNR of $5.5 \%$, accuracy of $8 \%$ based on compression ratio and accuracy of $0.9 \%$ based on maximum iteration than existing methods for enhancement on de-duplication and storage optimization. In the near future, more advanced optimization methods can be exploited, in order to highly enhance the de-duplication and storage optimization.

\begin{tabular}{|c|c|}
\hline \multicolumn{2}{|c|}{ Abbreviations } \\
\hline SOMI-GO & $\begin{array}{l}\text { Second Order Mutual Information based Grey } \\
\text { Wolf Optimization }\end{array}$ \\
\hline ED-PSO & $\begin{array}{l}\text { Euclidean Distance based Particle Swarm } \\
\text { Optimization }\end{array}$ \\
\hline ED-GA & Euclidean Distance based Genetic Algorithm \\
\hline ED-GWO & $\begin{array}{l}\text { Euclidean Distance based Grey Wolf } \\
\text { Algorithm }\end{array}$ \\
\hline IDC & International Data Company \\
\hline $\mathrm{CE}$ & Convergent Encryption \\
\hline $\mathrm{CDC}$ & Content-Defined Chunking \\
\hline RMD & $\begin{array}{l}\text { Resemblance and Mergence based De- } \\
\text { duplication scheme }\end{array}$ \\
\hline $\mathrm{AE}$ & Asymmetric Extremum \\
\hline HD & High Dimension \\
\hline
\end{tabular}

\section{References}

[1] Quinlan S and Dorward S 2002 Venti: a new approach to archival storage. In: Proceedings of USENIX FAST. https:// www.usenix.org/legacy/events/fast02/quinlan/quinlan_html/. Accessed 13 June 2007

[2] Xing Y, Xiao N, Liu F, Sun Z and He W 2015 AR-dedupe: an efficient de-duplication approach for cluster de-duplication system. J. Shanghai Jiaotong Univ. (Sci.) 20(1): 76-81

[3] He K, Huang C, Zhou H, Shi J, Wang X and Dan F 2015 Public auditing for encrypted data with client-side de-duplication in cloud storage. Wuhan Univ. J. Nat. Sci. 20(4): 291-298

[4] Kwon H, Hahn C, Kim D and Hur J 2017 Secure de-duplication for multimedia data with user revocation in cloud storage. Multimed. Tools Appl. 76(4): 5889-5903
[5] Zhang P, Huang P, He X, Wang H and Zhou K 2017 Resemblance and mergence based indexing for high performance data de-duplication. J. Syst. Softw. 128: 11-24

[6] Wang J, Zhao Z, Xu Z, Zhang H, Li L and Guo Y 2015 I-sieve: an inline high performance de-duplication system used in cloud storage. Tsinghua Sci. Technol. 20(1): 17-27

[7] Xia W, Jiang H, Feng D and Tian L 2014 Combining deduplication and delta compression to achieve low-overhead data reduction on backup datasets. In: 2014 Data Compression Conference, Snowbird, UT, pp. 203-212

[8] . Xia W, Jiang H, Feng D, Tian L, Fu M and Wang Z 2012 P-De-dupe: exploiting parallelism in data de-duplication system. In: 2012 IEEE Seventh International Conference on Networking, Architecture, and Storage, Xiamen, Fujian, pp. 338-347

[9] Bjørner N, Blass N and Gurevich Y 2010 Content-dependent chunking for differential compression, the local maximum approach. J. Comput. Syst. Sci. 76(3): 154-203

[10] Anand A, Muthukrishnan C, Akella A and Ramjee R 2009 Redundancy in network traffic: findings and implications. In: Proceedings of ACM SIGMETRICS

[11] Lu G, Jin Y and Du D H C 2010 Frequency based chunking for data de-duplication. In: 2010 IEEE International Symposium on Modelling, Analysis and Simulation of Computer and Tele Communication Systems, Miami Beach, FL, pp. 287-296

[12] Zhou B and Wen J 2013 Hysteresis re-chunking based metadata harnessing de-duplication of disk images. In: 2013 42nd International Conference on Parallel Processing, Lyon, pp. 389-398

[13] Bobbarjung D R, Jagannathan S and Dubnicki C 2006 Improving duplicate elimination in storage systems. ACM Trans. Storage (TOS) 2(4): 424-448

[14] Bonwick J 2009 ZFS De-duplication. https://blogs.oracle. com/bonwick/entry/zfsdedup. Accessed 11 Nov 2006

[15] Liu C, Xue Y, Ju D and Wang 2009 A novel optimization method to improve de-duplication storage system performance. In: 2009 15th International Conference on Parallel and Distributed Systems, Shenzhen, pp. 228-235

[16] Lu G, Nam Y J and Du D H C 2012 BloomStore: bloomfilter based memory-efficient key-value store for indexing of data de-duplication on flash. In: 2012 IEEE 28th Symposium on Mass Storage Systems and Technologies (MSST), San Diego, CA, pp. 1-11

[17] Liu C, Lu Y, Shi C, Lu G, Du D H C and Wang D S 2008 ADMAD: application-driven metadata aware de-duplication archival storage system. In: 2008 Fifth IEEE International Workshop on Storage Network Architecture and Parallel I/Os, Baltimore, MD, pp. 29-35

[18] Fu Y, Jiang H, Xiao N, Tian L, Liu F and Xu L 2014 Application-aware local-global source de-duplication for cloud backup services of personal storage. IEEE Trans. Parallel Distrib. Syst. 25(5): 1155-1165

[19] Li J, Li Y K, Chen X, Lee P P C and Lou W 2015 A hybrid cloud approach for secure authorized de-duplication. IEEE Trans. Parallel Distrib. Syst. 26(5): 1206-1216

[20] Kaczmarczyk M, Dubnicki C 2015 Reducing fragmentation impact with forward knowledge in backup systems with de-duplication. In: Proceedings of the 8th International Systems and Storage Conference(SYSTOR'15), IEEE, Haifa, Israel, pp. 201-208 
[21] Mirjalili S, Mirjalili S M and Lewis A 2014 Grey wolf optimizer. Adv. Eng. Softw. 69: 46-61

[22] Bhagwat D, Pollack K, Long D D, Schwarz T, Miller E L and Paris J-F 2006 Providing high reliability in a minimum redundancy archival storage system. In: Proceedings of IEEE MASCOTS

[23] Dubnicki C, Gryz L, Heldt L, Kaczmarczyk M, Kilian W, Strzelczak P, Szczepkowski J, Ungureanu C and Hydrastor M W 2009 A scalable secondary storage. In: Proceedings of USENIX FAST

[24] Yarrapragada K S S R and Krishna B B, Impact of tamanu oil-diesel blend on combustion, performance and emissions of diesel engine and its prediction methodology. J. Braz. Soc. Mech. Sci. Eng. 1-15

[25] Li X, Lillibridge M and Uysal M 2011 Reliability analysis of deduplicated and erasure-coded storage. ACM SIGMETRICS Perform. Eval. Rev. 38(3):4-9

[26] Haykin S 2005 Cognitive radio: brain-empowered wireless communications. IEEE J. Select. Areas Commun. 23: 201-220

[27] Cabric D, Mishra S M, Willkomm D, Brodersen R W and Wolisz A 2005 A cognitive radio approach for usage of virtual unlicensed spectrum. In: Proceedings of the 14th IST Mobile and Wireless Communications Summit, pp. 1-4

[28] Akyildiz I, Lee W, Vuran M and Mohanty S 2006 Next generation/dynamic spectrum access/cognitive radio wireless networks: a survey. Comput. Netw. 50: 2127-2159

[29] Tolic A and Brodnik A 2015 De-duplication in unstructured-data storage systems. Elektrotehniski Vestnik 82(5): 233-242

[30] Zhang Y et al 2017 A fast asymmetric extremum content defined chunking algorithm for data de-duplication in backup storage systems. IEEE Trans. Comput. 66(2): 199-211

[31] Barman S, Chattopadhyay S, Samanta D, Bag S and Show G 2014 An efficient fingerprint matching approach based on minutiae to minutiae distance using indexing with effectively lower time complexity. In: International Conference on Information Technology, Bhubaneswar, pp. 179-183

[32] Singh S, Bag S and Jenamani M 2015 Relative similarity based approach for improving aggregate recommendation diversity. In: Annual IEEE India Conference (INDICON), New Delhi, pp. 1-6

[33] Bag S, Tiwari M K and Chan F T S 2017 Predicting the consumer's purchase intention of durable goods: an attributelevel analysis. J. Bus. Res. (in press)

[34] Li X, Li J and Huang F 2016 A secure cloud storage system supporting privacy-preserving fuzzy de-duplication. Soft Comput. 20(4): 1437-1448

[35] Bellare M, Keelveedhi S and Ristenpart T 2013 Messagelocked encryption and secure de-duplication. Adv. CryptoEUROCRYPT 7881: 296-312

[36] Xia W et al 2016 A comprehensive study of the past, present, and future of data de-duplication. Proceedings of the IEEE 104(9): 1681-1710

[37] Eshghi K and Tang H K 2005 A framework for analyzing and improving content-based chunking algorithms. HewlettPackard Labs Technical Report TR, 30
[38] Kudova P 2007 Automatic clustering using genetic algorithms. In: 18th International Workshop on Database and Expert Systems Applications, IEEE Conference Publications, pp. $138-142$

[39] Kota P N and Gaikwad A N 2017 Optimized scrambling sequence to reduce paper. In space frequency block codes based MIMO-OFDM system. J. Adv. Res. Dyn. Control Syst. 502-525

[40] Bramhe S S, Dalal A, Tajne D and Marotkar D 2015 Glass shaped antenna with defected ground structure for cognitive radio application. In: International Conference on Computing Communication Control and Automation, Pune, pp. $330-333$

[41] Sunil Kumar B S, Manjunath A S and Christopher S 2016 Improved entropy encoding for high efficient video coding standard. Alex. Eng. J.

[42] Bhatnagar K and Gupta S 2017 Extending the neural model to study the impact of effective area of optical fiber on laser intensity. Int. J. Intell. Eng. Syst. 10(4): 274-283

[43] Balaji G N, Subashini T S and Chidambaram N 2015 Detection of heart muscle damage from automated analysis of echocardiogram video. IETE J. Res. 61(3), 236-243

[44] Gan G, Ng M K-P 2017 k-means clustering with outlier removal. Pattern Recognit. Lett. 90, 8-14

[45] Prasanna Kumar J and Govindharajulu P 2013 Near-duplicate web page detection: an efficient approach using clustering, sentence feature and fingerprinting. Int. J. Comput. Intell. Syst. 6(1): 1-13

[46] Manku G S, Jain A and Sarma A D 2007 Detecting nearduplicates. In: Russian Documents Through Using Fingerprint Algorithm Simhash

[47] Huffman coding and Huffman tree. www.csc.lsu.edu/ $\sim$ kundu/dstr/4-huffman.pdf

[48] Long W, Jiao J, Liang X and Tang M 2018 Inspired grey wolf optimizer for solving large-scale function optimization problems. Appl. Math. Model. 60, 112-126

[49] Gupta S and Deep K 2018 A novel Random Walk Grey Wolf Optimizer. Swarm Evol. Comput. https://doi.org/10.1016/j. swevo.2018.01.001

[50] Heidari A A and Pahlavani P 2017 An efficient modified grey wolf optimizer with Lévy flight for optimization tasks. Appl. Soft Comput. 60: 115-134

[51] Jiang F, Liu G, Du J and Sui Y 2016 Initialization of K-modes clustering using outlier detection techniques. Orig. Res. Article Inf. Sci. 332: 167-183

[52] Al Bahrani L T and Patra J C 2017 Orthogonal PSO algorithm for economic dispatch of thermal generating units under various power constraints in smart power grid. Appl. Soft Comput. 58: 401-426

[53] Nikolić M and Jović J 2017 Implementation of generic algorithm in map-matching model. Expert Syst. Appl. 72(15): 283-292

[54] Malhotra J and Bakal J 2018 Grey wolf optimization based clustering of hybrid fingerprint for efficient de-duplication. Multiagent. Grid. Syst. 14: 145-160 